Editorial

\title{
Correlating structure with visual function in patients with multiple sclerosis: Where is this leading?
}

\author{
See Article, pages $180-188$
}

Visual loss, particularly from optic neuritis (ON), is a major cause of disability in multiple sclerosis (MS). Determining the precise cause of the visual loss, what can be done to treat it and, indeed, what can be done to prevent it are major priorities in current clinical research. Over the last few decades, our understanding of the pathology in MS has shifted from a purely inflammationbased disease of axons in the white matter of the brain to a disorder which causes progressive degeneration of cells in the grey matter of the brain and in the ganglion cell layer of the retina. This has led to a reassessment of exactly how visual loss occurs in MS and a more detailed exploration of how structure and function might relate to each other.

At a 'macroscopic' level, there is an accepted understanding of the anatomy of the visual pathway from retina through the optic nerve, lateral geniculate nucleus, and optic radiations (OR) to the striate and extrastriate cortices. It is assumed that damage to this pathway is predictably associated with impairment of certain aspects of visual function, most notably visual fields, and this concept is fundamental to the clinical diagnostic process, i.e. that the pattern of visual field loss predicts the site of the lesion thanks to the preservation of retinal topography along the visual pathway. However, there are many other aspects of visual function which do not exhibit this tight correlation between structure and function. In particular, the association between structure and function at a more microscopic level remains unclear.

Nevertheless, the last two decades have witnessed an explosion of studies looking at how well abnormalities of visual function correlate with anatomical abnormalities of the visual pathways in MS. A major reason for the explosion is the continuing development and introduction of novel, and increasingly-sophisticated, ways of measuring both these parameters - every time a new technique is introduced its place as a research tool and its role in the clinic need to be established. This issue of Clinical Neurophysiology includes an article which examines the role of a newer measurement derived from optical coherence tomography (OCT), namely the thickness of the ganglion cell - inner plexiform layer (GCIPL) at the macula (Narayanan et al., 2019). OCT is a straightforward, widely available, quick, and relatively inexpensive test to perform, so it is natural to conclude that assessment of GCIPL thickness might prove useful in the clinical management of MS, but this depends on how well it reflects visual function.
It is worth exploring the reasons behind investigating the association between structure and function in more detail, and why researchers have invested so much in investigating this. At least three reasons spring to mind. First, these studies help to elucidate how normal visual function works: for over a century, lesion studies have played a crucial role in understanding normal visual physiology, and diseases such as MS provide a 'naturally-occurring' source of lesions. Second, they assist in understanding exactly what happens in disease to impair normal function, thereby shedding light on the disease process and what strategies might influence it. Third, an objective (anatomical) measurement might overcome the subjective variability which is inherent in the assessment of many aspects of visual function. This might, in turn, lead to a 'biomarker' for the disease, i.e. a measure which helps in the management of disease, either to assist with diagnosis, to determine prognosis, or to monitor the effects of therapeutic intervention. It is interesting to examine how the article by Narayanan et al. (2019) contributes to these different areas.

Visual function is complex, and no single measure can represent overall visual function. Individual aspects can be assessed in a number of different ways: for example, visual acuity can be determined by high- or low-contrast letter discrimination, visual fields can be studied using various different types of perimetry, and visual function at an electrophysiological level can be studied using electroretinography and visual evoked potentials (VEPs). The fact that VEPs are abnormal in eyes that have been affected by ON in MS has been known for nearly 50 years (Halliday et al., 1972). Indeed, VEPs once played a crucial role in the diagnosis of MS prior to the introduction of MRI scanning. The original assumption was that loss of amplitude of the VEP reflected an overall reduction in numbers of neurons while increased latency reflected loss of myelin.

It has been known for some time that multifocal VEPS (mfVEPs) identify damage in eyes of patients with MS that have not experienced optic neuritis (non-ON) (Laron et al., 2009; Ruseckaite et al., 2005). This does not appear to happen in the non-ON eyes of patients with Neuromyelitis Optica Spectrum Disorder (NMOSD) (Shen et al., 2018). The reason for the abnormalities of VEPs in nonON eyes in MS is not clear, but one possibility is that they arise largely as a result of damage in the optic radiations (OR) rather than the optic nerves. Graham and Klistorner (2017) have recently pointed out that, while the OR make up about $1 \%$ of total brain 
white matter, T2 lesions in the OR represent $7-10 \%$ of the total T2 lesion load in MS. In contrast, lesions of the OR are much less common in NMOSD, potentially explaining why non-ON eyes are not affected in this disorder (Shen et al., 2018). On the other hand, pattern electroretinogram studies in patients with glaucoma suggests that least some of the abnormality of mfVEP is explained by central GCIPL loss (Park et al., 2017), consistent with the strong association between OCT abnormalities and mfVEP amplitudes in patients with NMOSD (Shen et al., 2018). One possible synthesis might be that abnormalities of mfVEP latency are more related to OR damage while abnormalities of mfVEP amplitude are more related to retinal ganglion cell loss. Of course, the story may not be that simple: an intriguing suggestion is that some of the increased latency in non-ON eyes in MS might reflect physiological adaptation to damage in the contralateral, ON-affected, eye (Raz et al., 2013). For further background, readers should consult two recent reviews (Graham and Klistorner, 2017; Pihl-Jensen et al., 2017).

If some of the MfVEP abnormality does arise in the retina, a logical assumption is that this should be correlated with a reduction in retinal ganglion cell numbers. Changes in peripapillary retinal nerve fiber layer (pRNFL) thickness are thought to reflect axonal, and hence ganglion cell, loss. Accordingly, many studies have examined the correlation between the pRNFL and visual function in MS. More recently, the macular GCIPL thickness has been proposed as a better measure of ganglion cell loss because, as Narayanan et al. (2019) suggest, it may be "less confounded by gliosis and edema found in the RNFL". Recent meta-analyses of OCT studies suggest a marginally larger effect of MS on the pRNFL thickness compared to the GCIPL thickness in both ON and non-ON eyes (Britze et al., 2017; Petzold et al., 2018). Previous work has found that both pRNFL and GCIPL thickness decline over time in both ON and non-ON eyes (Narayanan et al., 2014) and several authors have reported correlations between macular ganglion loss and impaired visual function as assessed by both high- and lowcontrast visual acuity (Saidha et al., 2011; Seigo et al., 2012; Walter et al., 2012).

In this issue, Narayanan et al. (2019) bring all these factors together, looking at both ON and non-ON eyes of patients with MS: they compare both GCIPL and pRNFL thickness with low-contrast letter discrimination, macular perimetry, and amplitudes and latencies from mfVEPs. They confirm the high sensitivity of mfVEPs in the assessment of MS patients, in particular finding that more non-ON eyes showed an abnormality on mfVEP (45\%) than on OCT $(22 \%)(p<0.0005)$. An interesting feature of their analysis was a comparison of parameters of mfVEPs generated by stimulating central versus global visual fields. In their multiple-regression models (which controlled for covariates like age and MS-duration) mfVEP amplitude was significantly correlated with GCIPL thickness in both ON eyes ( $r=0.68$ centrally and 0.72 globally) and in nonON eyes $(r=0.46$ centrally and 0.48 globally, $p<0.0001$ for all). pRNFL thickness was somewhat less well-correlated with mfVEP amplitude $(\mathrm{r}=0.54)$ but only in ON eyes. Importantly, mfVEP latency was not correlated with either GCIPL or pRNFL thickness in these models, although it was in univariate analyses. Another recent study of non-ON eyes reported very similar correlations between mfVEP and both pRNFL and central ganglion cell layer thickness (Sriram et al., 2014).

Parenthetically, it is possible that modification of the mfVEP stimulus might lead to greater accuracy, and hence higher correlations. Narayanan et al. (2019) mention that more accurate mfVEP data might be obtained in response to so-called sparse mfVEP stimuli. Conventional mfVEP stimuli are very densely presented in time while sparse stimuli present transient onset stimuli interspersed between periods of no stimulation. This results in up to 15 times larger mfVEP responses, and 3-4 times the signal-to-noise ratios. Perhaps more importantly, the sparser the mfVEP stimuli the greater the proportion of non-ON eyes of MS patients that are discriminable from controls (Ruseckaite et al., 2005). The almost equal diagnostic power for ON and non-ON eyes obtained under those conditions has been verified in pupillographic studies that also employed multifocal sparse stimuli (Ali et al., 2014). The possible reasons behind these findings have been dealt with elsewhere (Maddess and Lueck, 2017).

To return to the three points mentioned earlier. What does this study contribute? Looking at the first two points, the findings are broadly consistent with the idea that the latency of mfVEPs reflects the integrity of the entire visual pathway, including (and, perhaps, especially) the OR, while amplitude of mfVEPs reflects the total number of ganglion cells in the retina. What about the third point? It is clear from the scatter plots in their figures 2 and 3 that there is a wide range in the absolute values of GCIPL and pRNFL thickness, and that the values straddle the cut-offs for their normative values. This is hardly surprising, considering the enormous inter-individual variation in normal subjects: the number of axons in human optic nerves can range from 0.78 to 1.68 million (Jonas et al., 1992), a factor of more than two, and the size of the LGN, OR and striate cortices vary by a factor of three (Andrews et al., 1997).

Similarly, the figures show that there is considerable overlap between the values of every parameter derived from ON and non-ON eyes. From a clinical perspective, this means that a single measurement of any of these parameters is unlikely to have useful positive predictive value regarding diagnosis of MS. A composite measure of pRNFL, GCIPL and mfVEP might do better in this regard but would be unlikely to supplant MRI as the major diagnostic tool.

On the other hand, mfVEP (or the proposed composite measure) might prove useful in prognostication and in studies of therapeutic interventions where any inter-individual baseline variability is no longer a confounding factor as patients are compared with themselves over time. Further work is clearly needed in this regard. The current study by Narayanan et al. (2019) cannot help with these clinical issues, but the process of comparing multiple parameters, namely mfVEP, OCT and psychophysical methods, may get us one step closer to the holy grail of a biomarker.

\section{Conflict of interest statement}

TM holds patents assigned to Konan Medical USA LLC, that refer to sparse multifocal evoked potential methods. CJL has no potential conflicts of interest.

\section{References}

Ali EN, Maddess T, James AC, Voicu C, Lueck CJ. Pupillary response to sparse multifocal stimuli in multiple sclerosis patients. Mult Scler 2014:20:854-61.

Andrews TJ, Halpern SD, Purves D. Correlated size variations in human visual cortex lateral geniculate nucleus, and optic tract. J Neurosci 1997;17:2859-68.

Britze J, Pihl-Jensen G, Frederiksen JL. Retinal ganglion cell analysis in multiple sclerosis and optic neuritis: a systematic review and meta-analysis. J Neurol 2017;264:1837-53. https://doi.org/10.1007/s00415-017-8531-y.

Graham S, Klistorner A. Afferent visual pathways in multiple sclerosis: a review. Clinical Exp Ophthalmol 2017;45:62-72. https://doi.org/10.1111/ceo.12751.

Halliday AM, McDonald WI, Mushin J. Delayed visual evoked response in optic neuritis. Lancet 1972;1:982-5.

Jonas JB, Schmidt AM, Muller-Bergh JA, Schlotzer-Schrehardt UM, Naumann GO. Human optic nerve fiber count and optic disc size. Invest Ophthalmol Vis Sci 1992;33:2012-8.

Laron M, Cheng H, Zhang B, Schiffman JS, Tang RA, Frishman LJ. Assessing visual pathway function in multiple sclerosis patients with multifocal visual evoked potentials. Mult Scler 2009;15:1431-41. https://doi.org/10.1177 1352458509350470.

Maddess T, Lueck CJ. Multiple sclerosis seen through new eyes. Editorial on Afferent visual pathways in multiple sclerosis: a review. Clin Exp Ophthal 2017;45:9-11. https://doi.org/10.1111/ceo.12885.

Narayanan D, Cheng H, Bonem KN, Saenz R, Tang RA, Frishman LJ. Tracking changes over time in retinal nerve fiber layer and ganglion cell-inner plexiform laye thickness in multiple sclerosis. Mult Scler 2014;20:1331-41. https://doi.org $\underline{10.1177 / 1352458514523498 .}$. 
Narayanan D, Cheng H, Tang RA, Frishman LJ. Multifocal visual evoked Potentials and contrast sensitivity correlate with ganglion cell-inner plexiform layer thickness in multiple sclerosis. Clin Neurophysiol 2019;130:180-8.

Park K, Kim J, Lee J. Measurement of macular structure-function relationships using spectral domain-optical coherence tomography (SD-OCT) and pattern electroretinograms (PERG). PLoS One 2017;12:e0178004. https://doi.org/ 10.1371/journal.pone.0178004.

Petzold A, Balcer LJ, Calabresi PA, Costello F, Frohman TC, Frohman EM, MartinezLapiscina EH, Green AJ, Kardon R, Outteryck O, Paul F, Schippling S, Vermersch P. Villoslada P, Balk LJ. Retinal layer segmentation in multiple sclerosis: a systematic review and meta-analysis. Lancet Neurol 2018;16:197-812.

Pihl-Jensen G, Schmidt MF, Frederiksen JL. Multifocal visual evoked potentials in optic neuritis and multiple sclerosis: a review. Clin Neurophysiol 2017;128:1234-45. https://doi.org/10.1016/i.clinph.2017.03.047.

Raz N, Chokron S, Ben-Hur T, Levin N. Temporal reorganization to overcome monocular demyelination. Neurology 2013;81:702-9. https://doi.org/10.1212 WNL.0b013e3182a1aa3e.

Ruseckaite R, Maddess T, Danta G, Lueck CJ, James AC. Sparse multifocal stimuli for the detection of multiple sclerosis. Ann Neurol 2005;57:904-13. https://doi org/10.1002/ana.20504.

Saidha S, Syc SB, Durbin MK, Eckstein C, Oakley JD, Meyer SA, Conger A, Frohman TC Newsome S, Ratchford JN, Frohman EM, Calabresi PA. Visual dysfunction in multiple sclerosis correlates better with optical coherence tomography derived estimates of macular ganglion cell layer thickness than peripapillary retinal nerve fiber layer thickness. Mult Scler 2011;17:1449-63. https://doi.org/ $10.1177 / 1352458511418630$.

Seigo MA, Sotirchos ES, Newsome S, Babiarz A, Eckstein C, Ford E, Oakley JD, Syc SB, Frohman TC, Ratchford JN, Balcer LJ, Frohman EM, Calabresi PA, Saidha S. In vivo assessment of retinal neuronal layers in multiple sclerosis with manual and automated optical coherence tomography segmentation techniques. J Neurol 2012;259:2119-30. https://doi.org/10.1007/s00415-012-6466-X.

Shen T, You Y, Arunachalam S, Fontes A, Liu S, Gupta V, Parratt J, Wang C, Barnett M, Barton J, Chitranshi N, Zhu L, Fraser CL, Graham SL, Klistorner A, Yiannikas C. Differing structural and functional patterns of optic nerve damage in multiple sclerosis and neuromyelitis optica spectrum disorder. Ophthalmology 2018. https://doi.org/10.1016/i.ophtha.2018.06.022.

Sriram P, Wang C, Yiannikas C, Garrick R, Barnett M, Parratt J, Graham SL, Arvind H, Klistorner A. Relationship between optical coherence tomography and electrophysiology of the visual pathway in non-optic neuritis eyes of multiple sclerosis patients. PLoS One 2014;9:e102546. https://doi.org/10.1371/journal. pone. 0102546 .

Walter SD, Ishikawa H, Galetta KM, Sakai RE, Feller DJ, Henderson SB, Wilson JA Maguire MG, Galetta SL, Frohman E, Calabresi PA, Schuman JS, Balcer LJ. Ganglion cell loss in relation to visual disability in multiple sclerosis. Ophthalmology 2012;119:1250-7. $\quad$ https://doi.org/10.1016/i. ophtha.2011.11.032.

Ted Maddess $*$

Eccles Institute for Neuroscience, John Curtin School of Medical Research, Bldg 131 Garran Road, Australian National University, Acton, ACT 2601,

Australia

* Corresponding author.

E-mail address: ted.maddess@anu.edu.au

Christian J. Lueck

Department of Neurology, The Canberra Hospital, PO Box 11, Woden, ACT 2606, Australia

Australian National University Medical School, Acton, ACT 2601, Australia

Available online 22 November 2018 